\title{
Multiple Nodular Lesions in Spleen and Liver in Visceral Leishmaniasis
}

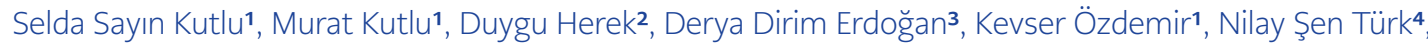 \\ Olga Yaylalı, ${ }^{5}$ Sibel Hacıoğlu6, Hüseyin Turgut \\ 1 Department of Infectious Diseases and Clinical Microbiology, Pamukkale University School of Medicine, Denizli, Turkey \\ 2 Department of Radiology, Pamukkale University School of Medicine, Denizli, Turkey \\ 3 Department of Parasitology, Ege University School of Medicine, İzmir, Turkey \\ 4 Department of Pathology, Pamukkale University School of Medicine, Denizli, Turkey \\ 5 Department of Nuclear Medicine, Pamukkale University School of Medicine, Denizli, Turkey \\ 6 Department of Hematology, Pamukkale University School of Medicine, Denizli, Turkey
}

\begin{abstract}
Objective: We aimed to describe the clinical, laboratory, and radiological characteristics of visceral leishmaniasis (VL) in our region to find additional clues that can help diagnosis.

Materials and Methods: All the patients with VL who admitted to our clinic between January 2010 and January 2018 were reviewed retrospectively. The patients with symptoms and signs suggestive of VL and a positive indirect fluorescent antibody test (IFA) or rapid diagnostic test, and/or direct parasitology consisting of the presence of amastigotes of Leishmania in bone marrow aspirate were included in the study. We collected the demographic and clinical features, laboratory, and radiological information from the hospital database.

Results: We present ten (seven males and three females) cases of VL, with the mean age of 53 (range of 31-75). Serological tests such as IFA or rapid diagnostic tests were positive in all patients. Amastigotes of Leishmania were present in only three of eight patients who underwent bone marrow biopsy. Abdominal magnetic resonance imaging or computed tomography revealed that all patients (10/10) had splenomegaly, eight of 10 patients had hepatomegaly, and seven patients had intraabdominal lymphadenopathy. Seven patients had multiple hypodense nodular lesions in the spleen with homogeneous parenchyma. Two patients had nodular lesions in the liver. Two patients died because of multiorgan failure before completing their treatment. The remaining patients completed liposomal amphotericin B treatment and cured.

Conclusion: VL should be suspected in the diagnosis of the patients, who had long-term fever, splenomegaly, pancytopenia associated with multiple nodular lesions in the spleen with/without liver involvement.

Keywords: Visceral leishmaniasis, spleen, multiple, nodular lesions, magnetic resonance imaging, computed tomography
\end{abstract}

\section{INTRODUCTION}

V isceral leishmaniasis (VL) is one of the important life-threatening infectious diseases worldwide (1). The highest prevalence of VL is in Brazil, East Africa and in South-East Asia, with an estimate of 50,000 to 90,000 new cases each year. VL is
Corresponding Author: Selda Sayın Kutlu

E-mail: sayinkutlu@yahoo.com

Received: June 11, 2019 Accepted: September 12, 2019 Published: October 7, 2019

\section{Suggested citation:}

Sayın Kutlu S, Kutlu M, Herek D, Erdoğan DD, Özdemir K, Şen Türk N, Yaylalı O, Hacıoğlu S, Turgut H. Multiple Nodular Lesions in Spleen and Liver in Visceral Leishmaniasis. Infect Dis Clin Microbiol 2019; 2: 70-77.

DOI: 10.36519/idcm.2019.19011 
an anthroponotic and zoonotic disease (2). While the anthroponotic VL occurs mainly in South Sudan and Sudan, zoonotic VL occurs across the Mediterranean basin (3). Sporadic VL is reported in Turkey, mostly in the Armenian border, Aegean, Central Anatolia, and Mediterranean regions. In 2016, 37 VL cases were reported from Turkey (4).

In newly endemic areas or in regions where sporadic cases have been reported, VL can be misdiagnosed leading to a significant delay in treatment (5). Over time mortality of VL increases if it is not treated (6). Therefore, early diagnosis is critical for the survival of VL cases. Delayed diagnosis is often because of the presence of non-specific symptoms, the low detection rate of amastigotes in bone marrow (BM) smears, and negative results on serological tests in the early stages of VL (7). So, there is a need for additional information or parameters that raise the suspicion of VL.

Abdominal imaging is frequently performed in patients with prolonged fever, pancytopenia, and hepatosplenomegaly. Although radiological signs of VL are non-specific, many patients with VL had pronounced hepatosplenomegaly and intraabdominal lymphadenopathy (6). Additionally, recent reports state that multiple hypodense nodules in the spleen are present which are superimposed to homogeneous parenchyma in abdominal computed tomography (CT) or magnetic resonance imaging (MRI) (6-10)

In this study, we described the presence of multinodular lesions in the spleen and/or liver in radiological imaging to detect additional clues that can help the diagnosis of visceral leishmaniasis (VL).

\section{HIGHLIGHTS}

- Visceral leishmaniasis (VL) is reported sporadically in Turkey.

- The meantime between symptom onset and diagnosis of VL is long.

- The diagnosis of VL is frequently difficult.

- VL should be suspected in diagnosis of the patients who had nodular lesions in the spleen with/without liver involvement.

\section{MATERIALS AND METHODS}

\section{Study population}

We retrospectively reviewed all the cases of VL between 2010 and 2018 in Pamukkale University Hospital in Denizli, Turkey. In these patients with symptoms and signs suggestive of VL, the diagnosis was made when serological titer is $\geq 1: 64$ in indirect fluorescent antibody (IFA) test or rapid immunochromatographic test is positive and/or amastigotes of Leishmania were present in BM aspirate stained by Giemsa stain. BM culture for Leishmania could not be performed.

We collected the demographic and clinical features, laboratory, and radiological information from the hospital database.

The IFA tests were performed in the serology laboratory of Parasitology Department in Ege University, İzmir, Turkey. The IFA IgG test was performed in the laboratory to investigate the presence of anti-Leishmania antibodies in the serum samples (11, 12). The rK39 rapid immunochromatographic test was performed in serum samples as recommended by the manufacturer.

All patients underwent abdominal CT or MRI. Also, some patients underwent abdominal ultrasonography or positron emission tomography-computed tomography (PET-CT). All CT examinations were done with a 16-detector CT scanner (Brilliance 16; Philips medical systems, Cleveland, OH, USA) from the lung bases to the pelvis. Computed tomography examinations were done after the injection of intravenous non-ionic contrast material on portal phase using the following parameters, collimation: $16 \times 1.5 \mathrm{~mm}$, pitch: 1.35 , tube potential: $120 \mathrm{kV}$ and reconstruction slice thickness: $3 \mathrm{~mm}$. All abdominal MRI examinations were performed with a 1.5 Tesla MRI system (Ingenia; Philips Medical Systems, Best, The Netherlands) using an eight-channel phased-array torso coil. Axial T2 turbo spin-echo (Time of repetition (TR): $1250 \mathrm{~ms}$; Time of echo (TE): 80ms, slice thickness: $6 \mathrm{~mm}$ ) and pre and post-contrast dynamic MR imaging with T1 weighted 3-dimensional imaging technique (TR: $3.6 \mathrm{~ms}$, TE: $1.65 \mathrm{MS}$ ) of the upper abdomen were acquired. Dynamic imaging of arterial, portal and late venous phases was done following intravenous contrast administration for each patient. 


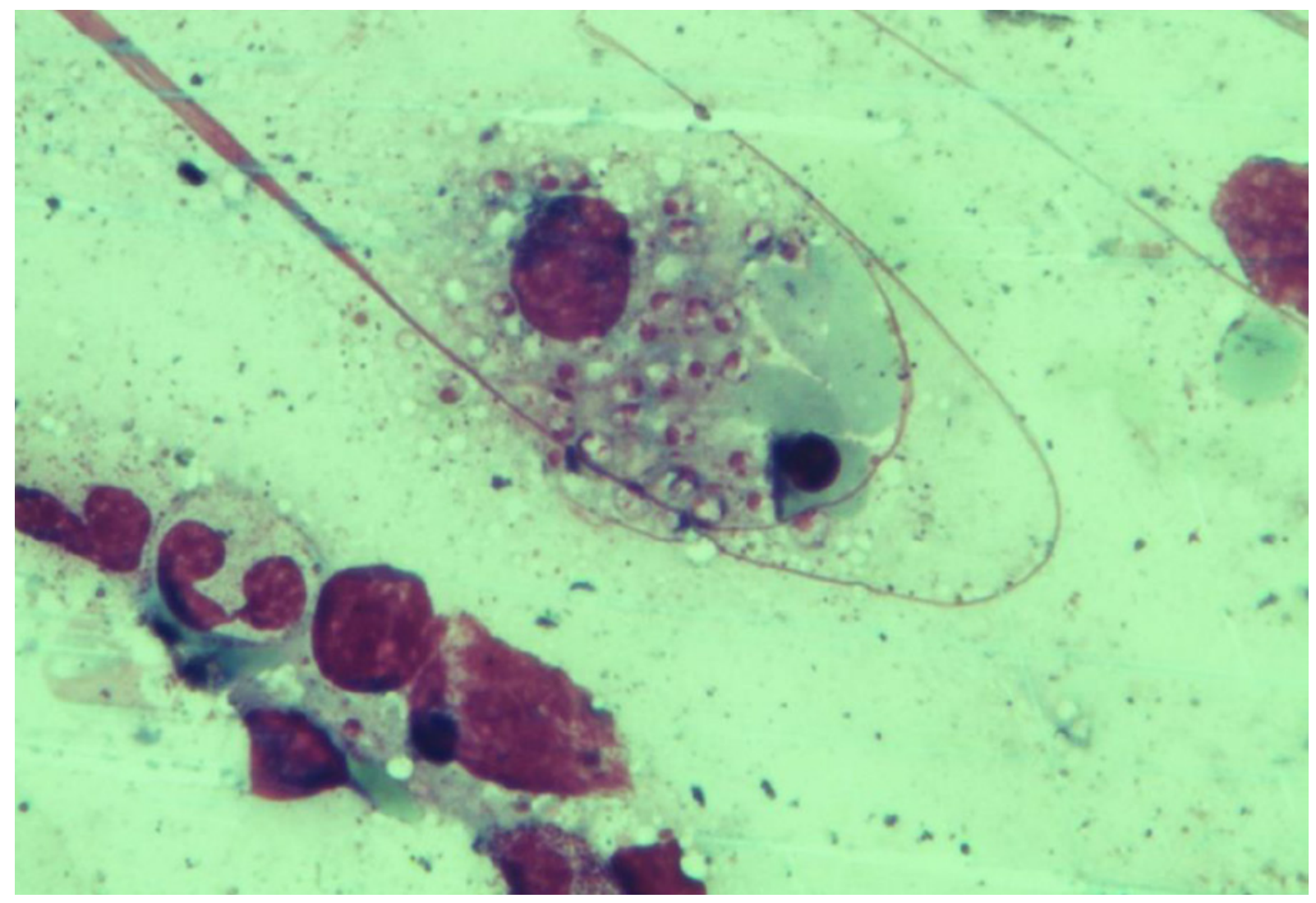

Figure 1. Patient 4: Bone marrow aspirate reveals macrophages with numerous nuclei of intracellular amastigotes of Leishmania parasites (Giemsa stain, 1000X).

All patients were treated with liposomal amphotericin B (L-AmB) $3 \mathrm{mg} / \mathrm{kg} /$ day for five days and on days 14 , and 21 .

\section{RESULTS}

We presented 10 (seven males and three females) cases of VL. The mean age was 53 (range of 31-75). The mean duration from the onset of the symptoms to diagnosis was 61 days (range: 22-120 days) (Table 1). IFA test was performed in seven patients, and all were positive. The rK39 rapid immunochromatographic test was positive in the other three patients. However, the fourth patient had the first rapid test negative; after three months, his IFA test was positive. Amastigotes of Leishmania were present in only three of eight patients who underwent a BM biopsy (Figure 1). The fourth patient's first BM aspirate had no amastigotes, after one month, Leishmania amastigotes were seen in second BM aspirate.
Six of the ten patients had no underlying diseases. Three of the remaining four patients had diabetes mellitus (DM), and one had prostate cancer in remission (Table 1).

All patients had fever, four patients had night sweats, and four patients had weight loss. There was no information about night sweats and weight loss among the remaining six patients (Table 1 ). There were two patients with peripheral lymphadenopathy.

All patients had leukopenia, nine patients had anaemia, and eight patients had thrombocytopenia. There was elevated lactate dehydrogenase in all patients, elevated liver enzymes in eight patients, and the presence of cholestasis (elevated gamma glutamate dehydrogenase and direct bilirubin) in seven patients. Seven patients had hypoalbuminemia, and four patients had hyperglobulinemia. All patients had elevated C-reactive protein (range: 3-15 $\mathrm{mg} / \mathrm{dL}$ ). Procalcitonin was measured in five patients 
Table 1. Characteristics of demographic, clinical, laboratory and radiological data of the patients with visceral leishmaniasis.

\begin{tabular}{|c|c|c|c|c|c|c|c|c|c|c|}
\hline \multirow{2}{*}{ Characteristics } & \multicolumn{10}{|c|}{ Patient } \\
\hline & 1 & 2 & 3 & 4 & 5 & 6 & 7 & 8 & 9 & 10 \\
\hline Age, year & 31 & 64 & 28 & 49 & 38 & 69 & 75 & 62 & 49 & 65 \\
\hline Gender & Male & Female & Male & Male & Male & Male & Female & Male & Female & Male \\
\hline Year & 2010 & 2010 & 2013 & 2015 & 2016 & 2017 & 2017 & 2018 & 2018 & 2018 \\
\hline Season & Winter & Spring & Summer & Spring & Autumn & Winter & Spring & Winter & Spring & Summer \\
\hline Underlying disease & None & $\mathrm{DM}^{\&}, \mathrm{HT}^{\prime \prime}$ & None & None & None & $\begin{array}{l}\text { Prostate } \\
\text { cancer }\end{array}$ & $\mathrm{DM}^{\&}, \mathrm{HT}^{\prime \prime}$ & None & None & $\mathrm{DM}^{\&}, \mathrm{HT}$ \\
\hline $\begin{array}{l}\text { The mean time between symptom } \\
\text { onset and hospital admission, days }\end{array}$ & 30 & 40 & 90 & 15 & 15 & 30 & 15 & 15 & 30 & 10 \\
\hline $\begin{array}{l}\text { The mean time between hospital } \\
\text { admission and the diagnosis, days }\end{array}$ & 15 & 20 & 5 & 90 & 7 & 90 & 30 & 8 & 47 & 15 \\
\hline Fever & Yes & Yes & Yes & Yes & Yes & Yes & Yes & Yes & Yes & Yes \\
\hline Night sweating & Yes & $U^{\beta}$ & Yes & Yes & Yes & $U^{\beta}$ & $U^{\beta}$ & $U^{\beta}$ & $U^{\beta}$ & $U^{\beta}$ \\
\hline Weight loss & Yes & $U^{\beta}$ & Yes & Yes & $U^{\beta}$ & Yes & $U^{\beta}$ & $U^{\beta}$ & $U^{\beta}$ & $U^{\beta}$ \\
\hline Leukocytes (/mm3) & 3110 & 3760 & 2500 & 1420 & 3000 & 3860 & 2000 & 3840 & 2770 & 1620 \\
\hline Neutrophils (/mm3) & 1540 & 2710 & 540 & 1100 & 1890 & 2000 & 1230 & 2290 & 2160 & 830 \\
\hline Lymphocytes (/mm3) & 971 & 880 & 1350 & 220 & 720 & 1000 & 580 & 1400 & 540 & 630 \\
\hline Hemoglobin (g/dL) & 11.9 & 10,3 & 10.6 & 8.5 & 14 & 10.5 & 10.5 & 9.7 & 7.5 & 9.40 \\
\hline Platelets (/mm3) & 143000 & 179000 & 107000 & 8000 & 154000 & 121000 & 49000 & 116000 & 24000 & 46000 \\
\hline C-reactive protein (mg/dL) & 4.8 & 7.0 & 3.2 & 16 & 5.1 & 3 & 6.5 & 12 & 15 & 5.6 \\
\hline Creatinine (mg/dL) & 0.9 & 0.9 & 1 & 0.5 & 1.2 & 1 & 0.5 & 0.9 & 0.5 & 1.2 \\
\hline Aspartate transaminase (IU/L) & 92 & 45 & 25 & 87 & 165 & 76 & 61 & 479 & 23 & 113 \\
\hline Alanine transaminase (IU/L) & 131 & 43 & 20 & 42 & 150 & 49 & 37 & 353 & 26 & 130 \\
\hline Lactate dehydrogenase (IU/L) & 475 & 479 & 227 & 921 & 521 & 622 & 320 & 1381 & 536 & 376 \\
\hline Alkaline phosphatase (IU/L) & 46 & $\mathrm{ND}^{\infty}$ & 51 & $\mathrm{ND}^{\infty}$ & 109 & 168 & 94 & 699 & 60 & 421 \\
\hline $\mathrm{GGT}^{\#}(\mathrm{IU} / \mathrm{L})$ & 71 & 68 & 20 & 94 & 165 & 160 & 82 & 671 & 18 & 536 \\
\hline Direct bilirubin (mg/dL) & 0.34 & 0.48 & 0.33 & 3.32 & 0.32 & 0.32 & 0.39 & 1.1 & 0.14 & 0.61 \\
\hline Albumin (g/dL) & 2.7 & 2.7 & 3.8 & 2.7 & 3.6 & 3.6 & 2.6 & 2.7 & 2.3 & 2.9 \\
\hline Globulin (g/dL) & 3.1 & 3.3 & 5.4 & 5.4 & 2.5 & 4.1 & 3.2 & 4.2 & 3.1 & 2.8 \\
\hline Albumin/globulin rate & 0.87 & 0.82 & 0.70 & 0.51 & 1.44 & 0.86 & 0.80 & 0.40 & 0.70 & 1.03 \\
\hline Prothrombin time & 12.9 & 14 & $\mathrm{ND}^{\infty}$ & 17.4 & 16.8 & 13 & 13 & $\mathrm{ND}^{\infty}$ & $\mathrm{ND}^{\infty}$ & 15.8 \\
\hline Ferritin (ng/dL) & 681 & 8325 & $\mathrm{ND}^{\infty}$ & 3286 & 956 & 1737 & 1528 & 2047 & 2000 & 989 \\
\hline Procalcitonin (ng/mL) & $N^{\infty}$ & $\mathrm{ND}^{\infty}$ & $\mathrm{ND}^{\infty}$ & 3 & $N^{\infty}$ & $N D^{\infty}$ & 1 & 3 & 12 & 2 \\
\hline Splenomegaly & Yes & Yes & Yes & Yes & Yes & Yes & Yes & Yes & Yes & Yes \\
\hline Hepatomegaly & Yes & Yes & Yes & Yes & Yes & No & Yes & No & Yes & Yes \\
\hline Multiple nodules in the spleen & Yes & Yes & No & No & Yes & Yes & Yes & Yes & No & Yes \\
\hline Multiple nodules in the liver & No & Yes & No & No & No & Yes & No & No & No & No \\
\hline Intraabdominal LAP* & Yes & Yes & No & No & Yes & No & Yes & Yes & Yes & Yes \\
\hline IFA positivity & $N P^{\epsilon}$ & $N P^{\epsilon}$ & $N P^{\epsilon}$ & $1 / 256$ & $1 / 512$ & $1 / 1024$ & $1 / 128$ & $1 / 256$ & $1 / 1024$ & $1 / 1024$ \\
\hline Rapid immunochromatographic test & Positive & Positive & Positive & Negative & $N P^{\epsilon}$ & $N P^{\epsilon}$ & $N P^{\epsilon}$ & $N P^{\epsilon}$ & $N P^{\epsilon}$ & $N P^{€}$ \\
\hline Seconder infection & No & No & No & $\mathrm{NE}^{\mu}$ & $\mathrm{NE}^{\mu}$ & $\mathrm{NE}^{\mu}$ & Sepsis, CC $^{\alpha}$ & Sepsis & Sepsis & $\mathrm{CC}^{\alpha}$ \\
\hline $\begin{array}{l}\text { Leishmania amastigotes in bone } \\
\text { morrow aspirate }\end{array}$ & None & None & Yes & Yes & Yes & No & No & No & No & No \\
\hline Outcome & Cure & Cure & Cure & Exitus & Cure & Cure & Cure & Cure & Cure & Exitus \\
\hline
\end{tabular}

\&: Diabetes mellitus, ": Hypertension, ${ }^{\beta}$ : Unknown, ${ }^{\infty}$ : No data, ${ }^{\#}$ : Gamma glutamate dehydrogenase

${ }^{*}$ : Lymphadenopathy, ${ }^{€}$ : Not performed, ${ }^{\mu}$ : No evidence, ${ }^{\alpha}$ : Cholecystitis 
and was $\geq 1 \mathrm{ng} / \mathrm{ml}$ in all. The elevation of ferritin (average $2394 \mathrm{ng} / \mathrm{ml}$ ) was observed in all patients except for one (Table 1).

Serology screening revealed negative results for acute cytomegalovirus, acute Epstein-Barr virus, and Human immunodeficiency virus (HIV) infections in all patients. No patients had evidence for lymphoma in BM histology. The laboratory and clinical criteria of hemophagocytic lymphohistiocytosis (HLH) were observed in the fourth patient and diagnosis of HLH was established (13). Secondary bacterial infection was found in four patients. There was sepsis in three patients; two of these patients had blood culture positive for Enterococcus faecalis and Cornybacterium striatum, the other one with cholecystitis. The other patient had cholecystitis without sepsis.

The seventh patient underwent to splenectomy to treat uncontrolled pancytopenia with unknown aetiology. At the time of splenectomy, she was hospitalized by the haematology clinic, and Leishmania amastigotes were not observed in her BM aspirate and the serological tests were not performed before. The Leishmania amastigotes were not observed either in the pathological examination of the spleen.

At the beginning of the course of the disease, four out of the 10 patients received steroids for the improvement of pancytopenia, with the suspect of rheumatic diseases. But, the negative results of rheumatologic tests and unimproved clinical status despite steroid therapy led us to diagnosis of VL.

Two of 10 patients died because of multiorgan failure before completing their VL treatment. One of the patients who received steroid and one fatal case had negative serological test results and no amastigotes in BM aspirate, which were performed in the first institution. However, one month later, a repeat $\mathrm{BM}$ aspirate and serological tests were performed and both proved the VL diagnosis. The remaining patients completed L-AmB treatment, recovered, and none of them relapsed until June 2019.

All the patients underwent abdominal CT or MRI, all had splenomegaly, eight had hepatomegaly, and seven had intraabdominal lymphadenopathy.

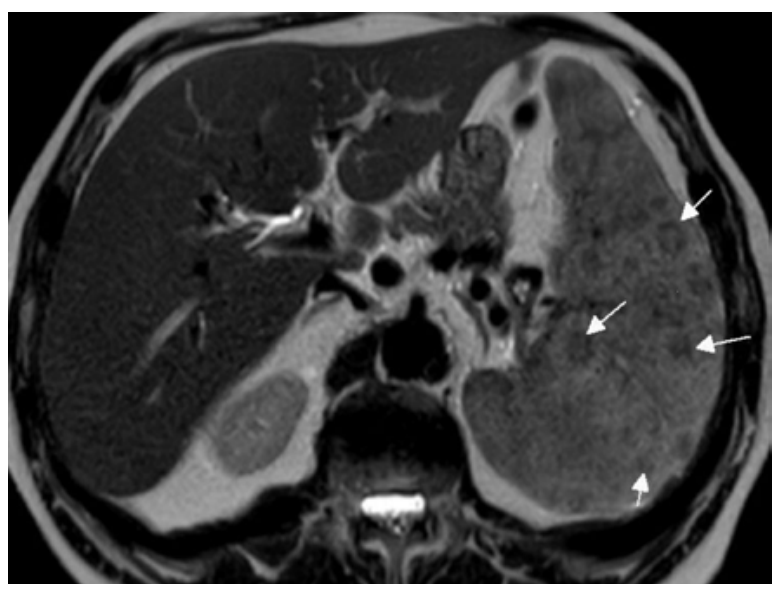

Figure 2. Patient 6: Magnetic resonance imaging T2 weighted sequence of the abdomen. Axial nonfat -saturated singleshot turbo spin-echo T2- weighted image shows prominent hypointense nodules throughout the spleen (white arrows).

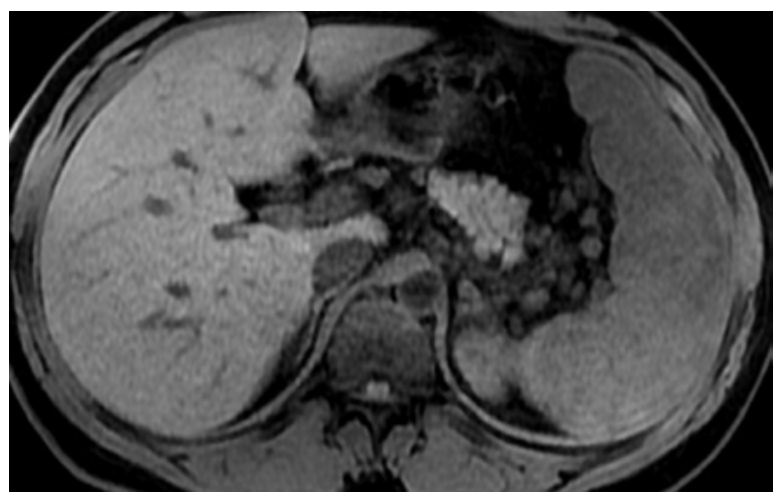

Figure 3. Patient 1: Magnetic resonance imaging T1 weighted sequence of the abdomen. Unenhanced axial T1-weighted fatsaturated 3 -dimensional image shows minimal heterogeneity of the spleen.

In seven of ten patients, there were multiple hypodense nodular lesions superimposed to homogeneous parenchyma in the spleen (Figure 2 and 3) Also, two of them had nodular lesions in the liver. Only four of 10 patients had control of imaging, and their radiological findings disappeared after treatment.

Only four patients had an abdominal ultrasound that showed splenomegaly in all, two of four had hypoechoic nodular lesions in the spleen, and three of four had hepatomegaly.

Because of unreasonable extensive investigations for the aetiology of fever, PET-CT was performed 
in two patients. In one of them (patient 4), PETCT showed hepatosplenomegaly with diffusely increased splenic Fluor 18 Fluorodeoxyglucose (F18 FDG) uptake ([standardized uptake value (SUV max:3.63). The seventh patient's PET-CT revealed hepatosplenomegaly with diffuse splenic slightly increased F-18 FDG uptake (SUV max: 1.77) with superimposed areas of multifocal hypodense nodules and diffusely increased F-18 FDG uptake (SUV max: 3.05-2.18) in BM areas of all the skeletal system.

\section{DISCUSSION}

We described the clinical, laboratory and radiological features of 10 cases with VL, which is sporadic in our region. Generally, there is a long duration from symptom onset until the diagnosis of VL like in our series $(6,14,15)$. We found that the mean duration between symptom onset and diagnosis was long, with over 60 days. Especially in one of our two patients who died, there were 90 days between the hospital admission and the diagnosis. Sometimes, an unnecessary splenectomy can be performed because of delayed diagnosis or misdiagnosis in patients with VL like as in our seventh patient (16). Sepsis, pneumonia, and jaundice are frequently concomitant with VL, especially in patients who have high parasitemia (17). Two patients had obvious cholestatic jaundice, and three patients had sepsis in our series. Therefore, early diagnosis is vital in cases with VL, to reduce death and complications.

Although most of our cases were immunocompetent, as previously reported, our four patients either had DM or solid malignancy that could be considered as immunosuppressed. However, these chronic conditions were not associated with late diagnosis or prognosis (18-23). In the previous reports, HIV infection was one of the most important risk factors for VL, although none of our patients was HIV-positive $(18-20,24)$. In our series, four patients received steroid treatment before the diagnosis of VL, and one of them resulted in death. We thought that steroid use might also have contributed to mortality, because immunosuppressive therapy may increase parasite burden and progression of the disease.

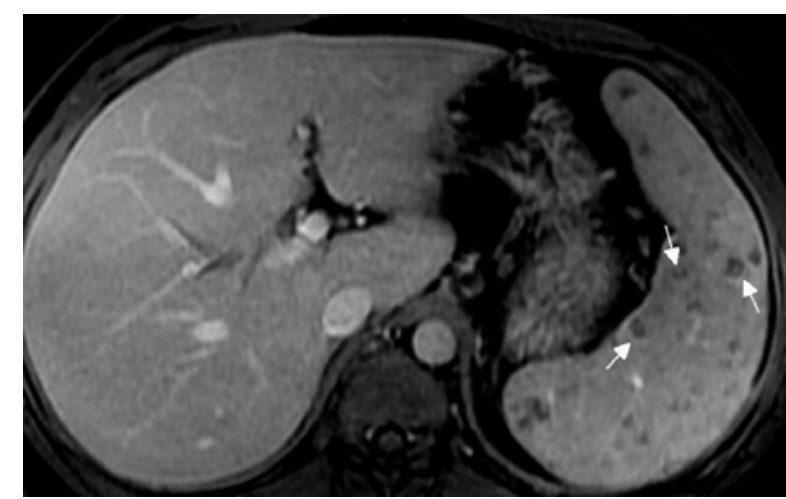

Figure 4. Patient 1: Magnetic resonance imaging $T 1$ weighted sequence of the abdomen. Following intravenous contrast media administration, many hypointense nodules are seen on portal phase, some with poor peripheral enhancement.

Although VL is seen in both genders, most of our cases were men, similar to the previous studies (15, 16, 24-26). Since men live outdoor more than women, they are exposed to phlebotomine sand flies which is a vector that causes VL transmission (25). Additionally, sex-linked biological factors such as sex hormone may affect immune responses to the parasites $(25,26)$.

Visceral leishmaniasis is a systemic disease affecting the reticuloendothelial system. In our series, the patients had fever, splenomegaly with/without hepatomegaly, pancytopenia, elevated liver enzymes, with similar frequency reported previously $(15,18$, 19). In previous reports, VL is found to be related to HLH $(14,27)$. In our series, HLH was diagnosed in only one patient; however, the other patients were not investigated for HLH. Although some of the laboratory and clinical criteria such as fever, splenomegaly, pancytopenia, and ferritin $\geq 500 \mu \mathrm{g} / \mathrm{dL}$ were present in most of our patients, we did not further investigate for HLH (13). Therefore, more patients might have HLH.

The diagnosis of VL can be frequently difficult due to the long incubation period and non-specific symptoms $(1,7)$. In sporadic regions, VL is not number one in the differential diagnosis list and specific diagnostic tests, such as detection of amastigotes in BM smears and serological tests may not be positive in the early period of the disease $(6,7)$. Although the serological tests were positive in all patients presented here, there was a delay 
in performing diagnostic tests in the ninth patient. Besides, in the fourth patient who was on steroid treatment and died, the first serological test (rK39 rapid immunochromatographic test) was negative, and amastigotes could not be demonstrated in the first BM aspirate. Except for a few publications in recent years, abdominal imaging is limited in the use for diagnosis of VL (7-9). During VL, the spleen is one of the most affected organs, and splenomegaly develops. Ultrasonography, CT and MRI detect multiple hypoechoic/hypodense nodules in the spleen. However, these images are non-specific and can be detected in septic emboli or abscesses, splenic infarction, metastatic disease, lymphoma, and granulomatous inflammatory diseases such as mycobacterial, fungal infections, cat-scratch disease, and sarcoidosis, as well as VL $(6,8-10,16)$. Most of our patients (7/10 patients) had multiple hypodense nodules in the spleen in which the parenchyma was usually homogeneous. Noticing this finding, although non-specific, associated with prolonged fever, pancytopenia, and splenomega- ly raised the suspicion of VL in our practice. Also, two of our patients had hypodense nodules in the liver. Previously similar cases with VL, which had enlarged liver and spleen with multiple hypoechoic, hypodense nodular lesions in abdominal tomography and ultrasonography, were also reported from Turkey (10). During the investigation of two cases with the fever of unknown origin, we performed PET-CT. There was an increased F-18 FDG uptake in the spleen (increased diffuse F-18 FDG uptake in the spleen both with and without superimposed hypodense nodules), similar to the patients in the literature $(28,29)$.

In conclusion, VL should be suspected among the patients, who had long-term fever, splenomegaly, pancytopenia associated with multiple nodular lesions in the spleen with/without liver involvement. According to our experience, although VL is not at the top of the differential diagnosis list, awareness and suspicion is the key to diagnose VL, especially in sporadic regions.
Peer-review: Externally peer-reviewed

Author Contributions: Concept - S.S.K., M.K., D.H., K.Ö.; Design - S.S.K., M.K., D.H., O.Y.; Supervision - H.T.; O.Y., S.S.K., M.K., S.H., N.Ş.T.; Materials - S.S.K., M.K., K.Ö., D.H.; Data Collection and/or Processing - S.S.K., D.D.E., N.Ş.T.; Analysis and/or Interpretation D.D.E., N.Ş.T., O.Y., H.T.; Literature Review - S.S.K., M.K.; Writer S.S.K., D.D.E.; Critical Reviews - All.
Conflict of Interest: The authors have no conflict of interest to declare.

Financial Disclosure: The authors declared that this study has received no financial support.

\section{REFERENCES}

1 Burza S, Croft SL, Boelaert M. Leishmaniasis. Lancet 2018; 392: 951-70. doi: 10.1016/S0140-6736(18)31204-2.

2 World Health Organisation (WHO). Leishmaniasis. 2019 March (cited 2019 May 20). Available from: URL: https://www.who.int/ en/news-room/fact-sheets/detail/leishmaniasis.

3 World Health Organisation (WHO). Leishmaniasis. 2019 (cited 2019 May 20). Available from: URL: http://www.emro.who.int/ health-topics/leishmaniasis/index.html

4 World Health Organisation (WHO). Leishmaniasis. Turkey Leishmaniasis Country Profiles. 2018 August (cited 2019 May 21). Available from: URL: https://www.who.int/leishmaniasis/ burden/Leishmaniasis Turkey/en/
5 Prestes-Carneiro LE, Spir PRN, Fontanesi M, Pereira Garcia KG, Silva FAD, Flores EF, et al. Unusual manifestations of visceral leishmaniasis in children: a case series and its spatial dispersion in the western region of São Paulo state, Brazil. BMC Infect Dis 2019; 19: 70. doi: 10.1186/s12879-018-3652-1.

6 Stojkovic M, Müller J, Junghanss T, Weber TF. Radiological Diagnoses in the Context of Emigration: Infectious diseases. Rofo 2018; 190: 121-33. doi: 10.1055/s-0043-120764.

7 Melchionda F, Varani S, Carfagnini F, Belotti T, Di Muccio T, Tigani R. Spleen nodules: a potential hallmark of Visceral Leishmaniasis in young children. BMC Infect Dis 2014; 14: 620. doi: 10.1186/s12879-014-0620-2 
8 Raeymaeckers S, Docx M, Demeyere N. MRI-findings of nodular lesions in an enlarged spleen, associated with visceral Leishmaniasis. Eur J Radiol. 2012; 81: 2550-3. doi: 10.1016/j. ejrad.2011.11.021.

9 Mao G, Yang G, Cheng Y, Zee CS, Huang W, Ni W, et al. Multiple nodular lesions in spleen associated with visceral leishmaniasis: a case report of MRI-findings. Medicine (Baltimore). 2014; 93: e272. doi: 10.1097/MD.0000000000000272.

10 Bukte Y, Nazaroglu H, Mete A, Yilmaz F. Visceral leishmaniasis with multiple nodular lesions of the liver and spleen: CT and sonographic findings. Abdominal Imaging 2004; 29: 82-4.

11 Daldal N, Taylan Özkan A. Parazit Kültürleri. Korkmaz M, Ok ÜZ (eds), Parazitolojide Laboratuvar. İzmir: META Basım; 2011.p.87-117.

12 Ylldız Zeyrek F, Dirim Erdogan D, Uluca N, Tumer S, Korkmaz M. 2012. Kutanöz Leishmaniasis Tanısında Serolojinin Yeri. Kaf kas Univ Vet Fak Derg 2012; 18 (Suppl-A): A121-4.

13 Henter JI, Horne A, Aricó M, Egeler RM, Filipovich AH, Imashuku S, et al. HLH-2004: Diagnostic and therapeutic guidelines for hemophagocytic lymphohistiocytosis. Pediatr Blood Cancer 2007; 48: 124-31.

14 Daher EF, Lima LL, Vieira AP, Nascimento LS, Soares DS, Abreu $\mathrm{KL}$, et al. Hemophagocytic Syndrome in Children with Visceral Leishmaniasis. Pediatr Infect Dis J 2015; 34: 1311-4. doi: 10.1097/INF.0000000000000916.

15 Varani S, Cagarelli R, Melchionda F, Attard L, Salvadori C, Finarelli AC, et al. Ongoing outbreak of visceral leishmaniasis in Bologna Province, Italy, November 2012 to May 2013. Euro Surveill 2013; 18: 20530.

16 Rinaldi F, Giachè S, Spinicci M, Corsi P, Ambu S, Gianfaldoni G. Focal spleen lesions in visceral leishmaniasis, a neglected manifestation of a neglected disease: report of three cases and systematic review of literature. Infection 2019; 47: 507 518. doi: 10.1007/s15010-019-01279-5.

17 Zacarias DA, Rolão N, de Pinho FA, Sene I, Silva JC, Pereira TC, et al. Causes and consequences of higher Leishmania infantum burden in patients with kala-azar: a study of 625 patients. Trop Med Int Health 2017; 22: 679-87. doi: 10.1111/tmi.12877.

18 Horrillo L, San Martín JV, Molina L, Madroñal E, Matía B, Castro A, et al. Atypical presentation in adults in the largest community outbreak of leishmaniasis in Europe (Fuenlabrada, Spain). Clin Microbiol Infect 2015; 21: 269-73. doi: 10.1016/j. cmi.2014.10.017.
19 Franceschini E, Puzzolante C, Menozzi M, Rossi L, Bedini A, Orlando $\mathrm{G}$, et al. Clinical and Microbiological Characteristics of Visceral Leishmaniasis Outbreak in a Northern Italian Nonendemic Area: A Retrospective Observational Study. Biomed Res Int 2016: 6481028. doi: 10.1155/2016/6481028.

20 Di Masi F, Ursini T, Iannece MD, Chianura L, Baldasso F, Foti G, et al. Five-year retrospective Italian multicenter study of visceral leishmaniasis treatment. Antimicrob Agents Chemother 2014; 58: 414-8. doi: 10.1128/AAC.00840-13.

21 Pagliano P, Carannante N, Gramiccia M, Ascione T, Stornaiuolo G, Gradoni L, et al. Visceral leishmaniasis causes fever and decompensation in patients with cirrhosis. Gut 2007; 56: 893-4.

22 Barbosa JF, de Figueiredo SM, Lyon S, Caligiorne RB. An 8-Year Retrospective Study of Human Visceral Leishmaniasis. Curr Clin Pharmacol 2016; 11: 265-9.

23 Ben Salem L, Khiari K, Turki S, Cherif L, Ali IH, Maazoun I, et al. [Obstructive acute renal failure revealing visceral leishmaniasis in a diabetic patient]. Nephrologie. 2003; 24: 95-9.

24 Sousa JMDS, Ramalho WM, Melo MA. Demographic and clinical characterization of human visceral leishmaniasis in the State of Pernambuco, Brazil between 2006 and 2015. Rev Soc Bras Med Trop 2018; 51: 622-630. doi: 10.1590/0037-8682-00472018.

25 Zheng C, Fu J, Li Z, Lin G, Jiang D, Zhou XN. Spatiotemporal Variation and Hot Spot Detection of Visceral Leishmaniasis Disease in Kashi Prefecture, China. Int J Environ Res Public Health 2018; 15. pii: E2784. doi: 10.3390/ijerph15122784.

26 Picado A, Ostyn B, Singh SP, Uranw S, Hasker E, Rijal S, et al. Risk factors for visceral leishmaniasis and asymptomatic Leishmania donovani infection in India and Nepal. PLoS One 2014; 9: e87641. doi: 10.1371/journal.pone.0087641.

27 Blázquez-Gamero D, Domínguez-Pinilla N, Chicharro C, Negreira S, Galán P, Pérez-Gorricho B, et al. Hemophagocytic lymphohistiocytosis in children with visceral leishmaniasis. Pediatr Infect Dis J 2015; 34: 667-9. doi: 10.1097/INF.0000000000000685.

28 Yapar AF, Reyhan M, Kocer NE, Aydin M, Nursal GN. Diffuse splenic F-18 FDG uptake in visceral leishmaniasis. Clin Nucl Med 2011; 36: 1041-3. doi: 10.1097/RLU.0b013e31821a2965.

29 Kyrtatos PG, Debard A, Martin-Blondel G, Alvarez M, Delobel P, Marchou B, et al. FDG-PET/CT findings during immune reconstitution in an HIV-1 patient infected with visceral leishmaniasis. Infection 2013; 41: 1017-9. doi: 10.1007/s15010-013-0459-2. 